\title{
Processamento Fonológico e Desempenho em Aritmética: Uma Revisão da Relevância para as Dificuldades de Aprendizagem
}

\author{
Júlia Beatriz Lopes Silva ${ }^{1}$ \\ Programa de Pós-Graduação em Saúde da Criança e do Adolescente da Universidade \\ Federal de Minas Gerais, Belo Horizonte, MG, Brasil \\ Laboratório de Neuropsicologia do Desenvolvimento da Universidade Federal \\ de Minas Gerais, Belo Horizonte, MG, Brasil \\ Ricardo José de Moura \\ Programa de Pós-Graduação em Neurociências da Universidade Federal de Minas Gerais, \\ Belo Horizonte, $M G$, Brasil \\ Laboratório de Neuropsicologia do Desenvolvimento da Universidade Federal \\ de Minas Gerais, Belo Horizonte, MG, Brasil \\ Guilherme Wood \\ Departamento de Neuropsicologia da Karl-Franzens University of Graz, Graz, Austria \\ Vitor Geraldi Haase \\ Departamento de Psicologia da Universidade Federal de Minas Gerais, \\ Belo Horizonte, $M G$, Brasil \\ Coordenação do Laboratório de Neuropsicologia do Desenvolvimento \\ da Universidade Federal de Minas Gerais, Belo Horizonte, MG, Brasil
}

\section{Resumo}

O processamento fonológico é tradicionalmente associado ao aprendizado da leitura e escrita. Seus componentes - consciência fonológica, memória fonológica e resgate lexical - são os principais preditores do desempenho na leitura e escrita e, déficits nestes domínios estão relacionados com a dislexia. Recentemente, a relação entre a fonologia e a matemática também tem sido demonstrada na literatura. O processamento fonológico está correlacionado, principalmente, com aspectos simbólicos da matemática, como a automatização de fatos aritméticos, resolução de problemas matemática e transcodificação numérica. Os diversos aspectos do processamento simbólico influenciam na aprendizagem dos dígitos e rótulos verbais que representam as magnitudes. Além disso, déficits destes domínios também ocasionam déficits na matemática, como é o caso de crianças disléxicas que também apresentam prejuízos na memorização da tabuada. Analisamos os principais artigos que abordam este tema, suas falhas metodológicas e principais contribuições teóricas. Sugerimos que o processamento fonológico é o principal mecanismo compartilhado entre a discalculia e a dislexia e que se relaciona à comorbidade entre os dois transtornos.

Palavras-chave: Processamento fonológico, discalculia, dislexia, comorbidade.

Endereço para correspondência: Universidade Federal de Minas Gerais, Faculdade de Filosofia e Ciências Humanas, Laboratório de Neuropsicologia do Desenvolvimento, sala 2016, Avenida Antônio Carlos, 6627, Pampulha, Belo Horizonte, MG, Brasil 31270-901. Fone: (31) 3409-6295/ 9683-3170. E-mail: juliablsilva@ gmail.com 


\title{
Phonological Processing and Mathematic Performance: A Review of the Relevance to Learning Disabilities
}

\begin{abstract}
The phonological processing is traditionally associated with learning to read and write. Its components phonological awareness, phonological memory and lexical access - are the main predictors of performance in reading and writing and, deficits in these areas are related to dyslexia. Recently, the relationship between the phonology and mathematics has also been demonstrated in the literature. The phonological processing is mainly correlated with the symbolic aspects of mathematics, such as the automation of arithmetic facts, arithmetic problem solving and numerical transcoding. The various aspects of symbolic processing influence the learning of digits and verbal labels that represents magnitudes. In addition, its deficits can also cause impairment in mathematics, such as dyslexic children who also showed worse automation of the multiplication table. We analyze the main articles that address this issue, its methodological flaws and major theoretical contributions. We suggest that phonological processing is the main mechanism shared between dyscalculia and dyslexia, and that it is related to the comorbidity between two disorders.
\end{abstract}

Keywords: Phonological processing, dyslexia, dyscalculia, comorbidity.

\section{Procesamiento Fonológico y el Rendimiento de la Aritmética: Una Revisión de la Importancia para las Dificultades de Aprendizaje}

\section{Resumen}

El procesamiento fonológico se asocia tradicionalmente a aprender a leer y escribir. Sus componentes, la conciencia fonológica, la memoria fonológica y el acceso al léxico - son los principales predictores del desempeño en lectura y escritura y, deficiencias en estas áreas están relacionadas con la dislexia. Recientemente, la relación entre la fonología y matemáticas también se ha demostrado en la literatura. El procesamiento fonológico está principalmente relacionado con los aspectos simbólicos de las matemáticas, tales como la automatización de los hechos aritméticos, resolución de problemas aritméticos y de transcodificación numérica. Los diversos aspectos de procesamiento simbólico influyen en el aprendizaje de dígitos y etiquetas verbales que representan las magnitudes. Además, estos déficits también causan um déficiti en las matemáticas, como los niños disléxicos que también mostraron una menor retención de la tabla de multiplicar. Se analizan los principales artículos que se ocupan de este tema, sus deficiencias metodológicas y las principales contribuciones teóricas. Se sugiere que el procesamiento fonológico es el principal mecanismo compartido entre discalculia y dislexia, y que se relaciona con la comorbilidad entre ambos trastornos.

Palabras clave: Procesamiento fonológico, dislexia, discalculia, comorbilidad.

Sabemos que existe comorbidade entre transtornos da aprendizagem da matemática e transtornos do desenvolvimento da linguagem oral e da aprendizagem da leitura e escrita; nos quais o processamento fonológico desempenha um papel importante (Landerl \& Moll, 2010; Simmons \& Singleton, 2008).

Qual é a natureza dessa conexão? Trata-se apenas de uma comorbidade, ou seja, co-ocor- rência aleatória? $\mathrm{Ou}$ existe algum compartilhamento de mecanismos? Há evidências de que vários mecanismos podem estar envolvidos na gênese das dificuldades de aprendizagem, tais como déficits na acuidade numérica não-simbólica e na memória de trabalho. Será que déficits no processamento fonológico desempenham algum papel nas dificuldades de aprendizagem da matemática? 
Para responder a estas questões, apresentamos uma revisão dos estudos sobre a importância do processamento fonológico para a aprendizagem da matemática em crianças típicas, bem como das evidências para o envolvimento de déficits no processamento fonológico nas dificuldades de aprendizagem de aritmética de crianças com SLI (specific language impairment), dislexia e discalculia. Trata-se de uma revisão não sistemática e abrangente da literatura. Constitui-se, portanto, de uma revisão narrativa, baseada na escolha arbitrária de tópicos relevantes para a área e na análise crítica da metodologia e resultado destes trabalhos. Todos os artigos encontram-se indexados no banco de dados PUBMED. Inicialmente, definiremos discalculia e dislexia e aspectos relacionados à comorbidade dos transtornos. Em seguida, analisaremos a contribuição específica de cada componente do processamento fonológico na matemática, correlatos anátomo-funcionais, e perfil de processamento numérico com crianças com transtornos de linguagem.

\section{Discalculia do Desenvolvimento}

A discalculia do desenvolvimento (DD) é um transtorno na aquisição de habilidades aritméticas básicas, relacionadas a déficits na representação de numerosidade ou magnitude, no reconhecimento e produção de numerais em suas diversas notações bem como de operadores aritméticos, na capacidade de aprender e resgatar automaticamente os fatos aritméticos, e na capacidade de realizar as quatro operações aritméticas (Butterworth, 2005; Geary, 2000; Shalev, Manor, \& Gross-Tsur, 1997).

A prevalência da discalculia varia entre 3 a $6 \%$ da população em idade escolar e sua origem é genética (Shalev, Auerbach, Manor, \& Gross-Tsur, 2000). O diagnóstico exige que as dificuldades sejam persistentes e não possam ser atribuíveis à deficiência mental, deficiências sensoriais, falta de estimulação ou experiência, métodos ou experiências educacionais inadequados ou a transtornos emocionais e motivacionais (American Psychiatric Association, 2000; Butterworth, 2005). A DD precisa ser diferenciada do baixo rendimento em matemática, uma disciplina notoriamente considerada difícil e que provoca ansiedade em um contingente apreciável da população (Lee, 2009). Os prejuízos exibidos pelas crianças com DD são graves e amplos, não se limitando apenas à execução de cálculos aritméticos. (Geary, Hoard, Byrd-Craven, \& DeSoto, 2004; Girelli \& Seron, 2001). Essas crianças apresentam dentre outras, dificuldades em associar representações internas de magnitude e numerais arábicos (Landerl, Bevan, \& Butterworth, 2004); achados neurofuncionais apóiam esta conexão entre discalculia do desenvolvimento e déficits no processamento básico de informação numérica (Rubinsten \& Henik, 2006).

A matemática é um domínio complexo que envolve diversas habilidades, como contagem, estimação e compreensão de procedimentos. Os principais mecanismos cognitivos subjacentes, candidatos para explicar as dificuldades apresentadas por crianças com discalculia são: o senso numérico (Dehaene, 1992), o processamento viso-espacial (Rourke \& Conway, 1997), a memóra de trabalho (Geary, 1993, 2010), e o processamento fonológico (Simmons \& Singleton, 2008).

O modelo cognitivo que atualmente melhor explica o processamento numérico é o modelo do código triplo (Dehaene, 1992), o qual propõe três tipos de representações de magnitude: analógica, arábico e verbal. A representação analógica está relacionada à ideia de senso numérico, sistema pré-verbal de raciocínio aritmético. Esta representação não-simbólica de magnitude relaciona-se com a compreensão do significado dos números, noção de quantidade e capacidade de estimação. De acordo com esse modelo, as representações analógicas constituem o conteúdo semântico para as representações simbólicas, arábicas e verbais, as quais constituem aquisições desenvolvimentais mais tardias, tanto filogeneticamente quanto ontogenicamente. No que diz respeito ao substrato neuroanatômico, a representação analógica relaciona-se principalmente com porções bilaterais do sulco intraparietal (Dehaene, Piazza, Pinel, \& Cohen, 2003). O processamento no sistema arábico (p. ex., 45) 
recruta bilateralmente uma rede neuronal cujo epicentro é o giro fusiforme (Dehaene \& Cohen, 1995), sendo importante para a realização de cálculos que envolvem algarismos com vários dígitos. O sistema verbal (p. ex., quarenta e cinco) é fundamentado na linguagem e possibilita a realização de cálculos exatos e problemas apresentados oralmente, tendo o giro angular esquerdo como seu epicentro (Zamarian, Ischebeck, \& Delazer, 2009).

Estudos recentes sugerem que crianças com discalculia teriam dificuldade com a manipulação de magnitudes não-simbólicas (Landerl, Fussenegger, Moll, \& Willburger, 2009; Mussolin, Mejias, \& Noël, 2010) e, de modo correlato, apresentariam uma menor acuidade na representação analógica de magnitudes (Piazza et al., 2010). De acordo com Halberda, Mazzoco e Feigenson (2008), diferenças individuais em testes padronizados de matemática podem ser explicadas por diferenças na acuidade do senso numérico.

O processamento viso-espacial influencia o desempenho na matemática principalmente porque se relaciona à habilidade da criança alinhar os números de forma adequada na hora de montar uma operação, ou manipular quantidades na memória de curto-prazo (Geary, 1993; Rourke \& Conway, 1997). Zuber, Pixner, Moeller e Nuerk (2009) encontraram correlações significativas $(r=-0,22, p<0,05)$ entre erros sintáticos de transcodificação numérica e memória viso-espacial (medida pela tarefa de Cubos de Corsi). A amostra utilizada neste estudo era composta de crianças austríacas de 7 anos, as quais utilizam uma regra de inversão na transcodificação numérica (por exemplo: o número 42 é lindo como dois e quarenta), o que pode justificar a correlação com a memória viso-espacial.

Estudos longitudinais sugerem que o processamento viso-espacial é crucial nos primeiros anos de aprendizagem da matemática para a representação de modelos mentais de quantidade que não envolvam rótulos verbais (Huttenlocher, Jordan, \& Levine, 1994; Rasmussen \& Bisanz, 2005). De acordo com LeFevre et al. (2010), a memória de trabalho viso-espacial é preditora independente do desempenho na matemática em crianças de 4 a 8 anos. A medida de atenção viso-espacial usada pelos autores, análoga à tarefa de Cubos de Corsi, correlacionou-se com medidas simbólicas e não-simbólicas do processamento numérico (leitura de números e comparação de magnitudes) nas crianças pré-escolares. Os autores sugerem que essa relação pode ser mediada pela circuitaria parietal, a qual está envolvida no processamento de magnitudes, assim como no processamento viso-espacial.

Geary (1993) sugere que as dificuldades de aprendizagem matemáticas estariam relacionadas a déficits na memória de trabalho. Segundo o autor, existiriam três subtipos de dificuldades de aprendizagem matemáticas, todas relacionadas a déficits de memória: subtipo procedimental, em que os déficits estariam associados à prejuízos na memória de trabalho verbal; subtipo relacionado à memória semântica, em que a criança apresentaria déficits na recuperação dos fatos aritméticos, e subtipo viso-espacial, em que o indivíduo apresentaria déficits na representação espacial de número.

Noël (2009) examinou a influência específica dos mecanismos da memória no processamento numérico de crianças pré-escolares. $\mathrm{O}$ executivo central foi o melhor preditor do desempenho, seguido pela memória de trabalho viso-especial.

Em um artigo de revisão recente, Raghubar, Barnes e Hetch (2010) reportaram o efeito robusto da memória de trabalho fonológica (especialmente testes que envolvem estímulos numéricos, como o Dígitos do WISC) na matemática. A memória de trabalho viso-espacial, segundo os autores, tem uma relação menos consistente com a matemática.

Além disso, a habilidade de contagem nos dedos prediz o desempenho na matemática e diferenças individuais: utilizar os dedos para representar quantidades diminui a sobrecarga da memória de trabalho, facilita a transição entre representações simbólicas e não-simbólicas e é uma habilidade prejudicada em crianças com discalculia (Costa et al., 2011). Os déficits na memória não explicam, entretanto, a especificidade dos prejuízos na aritmética (Dehaene, 1992). 
A consciência fonológica refere-se a habilidades de manipulação dos sons das palavras e é um dos principais preditores do desempenho na leitura (Vellutino, Fletcher, Snowling, \& Scanlon, 2004). Estudos recentes estabelecem um paralelo entre a ativação do giro angular esquerdo em tarefas de leitura e tarefas matemáticas que se baseiam em códigos verbais - transcodificação numérica, multiplicação - de acordo com o modelo do código triplo (De Smedt, Taylor, Archibald, \& Ansari, 2010; Simmons \& Singleton, 2008). Esses estudos demonstram a relevância do processamento fonológico principalmente em aspectos verbais da aritmética, como a multiplicação e frações. Para resolver estes problemas aritméticos, a criança deve converter os termos em um código verbal, processar essa informação fonológica e resgatar uma resposta da memória de longo prazo verbal.

\section{Comorbidade entre Discalculia e Transtornos da Linguagem}

Estudos epidemiológicos apontam altas taxas de comorbidade entre discalculia e dislexia: aproximadamente $40 \%$ dos disléxicos também apresentam déficits na cognição aritmética (Lewis, Hitch, \& Walker, 1994) e a prevalência de déficits na leitura e na aritmética é semelhante, por volta de 4 a 7\% (Landerl et al., 2009). Alguns estudos sugerem taxas de comorbidade de até $70 \%$, valor que pode ser superestimado, dependendo de critérios diagnósticos e dos construtos avaliados pelos testes padronizados de aritmética e leitura (Landerl \& Moll, 2010). A comorbidade entre discalculia e dislexia é maior do que seria esperada pelo acaso, se as duas entidades fossem totalmente segregadas de forma independente. Ao mesmo tempo, a comorbidade é relativamente específica, uma vez que numa família cujo caso índice é de discalculia, a probabilidade maior é de que ocorra recorrência para discalculia do que para dislexia. Além disso, questionários respondidos por pais sugerem que a transmissão é específica para cada transtorno e que dificuldades de leitura e matemática apresentam um padrão de co-segregação (Landerl \& Moll, 2010).
A dislexia do desenvolvimento caracteriza-se por um déficit específico na aquisição da leitura e acomete cerca de 5 a $17 \%$ de crianças em idade escolar (Shaywitz \& Shaywitz, 2005). De acordo com a World Health Organization, a International Dyslexia Association e o National Institute of Child Health dos Estados Unidos, a explicação proeminente em relação às dificuldades apresentadas pelos disléxicos é referente ao déficit central do processamento fonológico. Alguns autores, como Wimmer e Schurz (2010) criticam esse posicionamento devido à hegemonia de pesquisas na língua inglesa, que possui uma ortografia irregular. De acordo com Wimmer (2006), as altas taxas de comorbidade entre discalculia e dislexia em idiomas com ortografia mais transparente, como o alemão, podem estar relacionadas com dificuldades de fluência, já que crianças disléxicas alemãs têm como melhor preditor da leitura o desempenho na tarefa de nomeação rápida de estímulos visuais em detrimento do déficit na consciência fonológica. Outras teorias alternativas para a explicação dos déficits da dislexia incluem a hipótese do processamento temporal (Tallal, Miller, Jenkins, \& Merzenich, 1997), hipótese cerebelar (Fawcett \& Nicolson, 2001; Nicolson \& Fawcett, 2001) e hipótese do duplo déficit (Bowers \& Wolf, 1993; Wolf \& Bowers, 1999; Wolf, Bowers, \& Biddle, 2000). Entretanto, nenhuma dessas teorias descarta por completo a dificuldade de crianças disléxicas em tarefas fonológicas (Simmons \& Singleton, 2008).

Atualmente, duas hipóteses são proeminentes em relação à especificidade dos déficits cognitivos no caso da comorbidade discalculia/dislexia. Butterworth (2005), Landerl et al. (2004) e Wilson e Dehaene (2007) propõem a hipótese do déficit cognitivo específico de domínio (domain-specific cognitive deficit account), segundo a qual prejuízos no módulo numérico, ou no senso numérico, e não em habilidades gerais, como a memória, causariam dificuldades na aritmética. A dislexia, de modo análogo, seria causada especificamente por um déficit no processamento fonológico e o senso numérico estaria intacto em muitos casos. O senso numérico seria então o endofenótipo implicado nas 
dificuldades da matemática e o processamento fonológico, na leitura.

Landerl et al. (2004) estudaram crianças de 8 e 9 anos que foram categorizadas como 'dificuldade de leitura', 'dificuldade na matemática', 'dificuldade de leitura e matemática' e 'grupo controle'. Segundo os autores, crianças com dificuldades apenas na leitura apresentam desempenho normal em tarefas de processamento numérico, o que apoia a hipótese de que déficits fonológicos não influenciam a aritmética. Entretanto, é possível apontar uma falha metodológica no artigo, já que as crianças que foram classificadas como 'dificuldade na matemática' ou 'dificuldade de leitura e matemática' tiverem desempenho três desvios-padrão abaixo da média em um teste normatizado de aritmética. Em contrapartida, as crianças eram classificadas como 'dificuldade de leitura' quando obtinham desempenho abaixo do percentil $25 \mathrm{em}$ um teste de leitura, o que sugere que as crianças dos outros grupos tinham dificuldades mais graves.

Landerl et al. (2009) sugerem que discalculia e dislexia seriam transtornos com perfis neuropsicológicos diferentes e duplamente dissociados. Neste estudo, as pesquisadores avaliaram crianças entre 8 e 10 anos que foram classificadas como controles, disléxicas, discalcúlicas ou disléxicas e discalcúlicas em relação ao processamento numérico básico, consciência fonológica, memória de trabalho e velocidade de processamento. Segundo as autoras, os déficits encontrados no grupo duplo-déficit teriam caráter aditivo, e não, interativo, o que sugere que discalculia e dislexia são entidades nosológicas específicas.

Além disso, estudos de caso (Tressoldi, Rosati, \& Lucangeli, 2007) também sugerem uma dupla dissociação entre discalculia e dislexia: a comorbidade entre elas seria consequência da co-ocorrência dos déficits específicos a cada uma, a saber: déficits no processamento fonológica na dislexia e déficits no senso numérico na discalculia.

Por outro lado, a hipótese do déficit comum (commom deficit account; Vellutino et al., 2004) sugere que a discalculia seria, de alguma forma, causada pelos déficits fonológicos associados à dislexia. Esse pressuposto seria válido para as crianças com quadro comórbido de discalculia e dislexia, já crianças que apresentam apenas dificuldade na matemática teriam outro perfil cognitivo. Simmons e Singleton (2008), em um artigo de revisão sobre representações fonológicas e aritmética, apoiam essa hipótese ao sugerir que o processamento fonológico é preditor do desempenho aritmético e que, crianças com dislexia teriam representações fonológicas pobres que causam impacto negativo na aprendizagem da matemática (weak phonological representation hypothesis).

Jordan (2007) considera que as dificuldades de leitura agravam, e não causam, dificuldades na matemática, já que crianças com dislexia/discalculia teriam dificuldades em utilizar os mecanismos compensatórios associados à linguagem. Em seu artigo de revisão, a autora conclui que as crianças com dificuldade de matemática, independentemente da associação com dificuldades de leitura, apresentam déficits no senso numérico. O que diferencia o grupo discalculia e discalculia/dislexia é o fato que o grupo com duplo déficit apresenta maiores dificuldade na resolução de problemas matemáticos, os quais dependem, simultaneamente, da representação verbal e processamento numérico.

\section{Aprendizagem da Aritmética e Processamento Fonológico}

\section{Componentes do Processamento Fonológico e sua Relação Específica com a Matemática}

O desempenho de crianças em tarefas diversas de processamento fonológico depende da qualidade das suas representações dos códigos fonológicos (Simmons \& Singleton, 2008; Snowling \& Hulme, 1994). De acordo com Wagner e Torgesen (1987), os principais componentes do processamento fonológico associados à aquisição da leitura são os seguintes: resgate lexical, memória de trabalho/ memória de curto prazo fonológica e consciência fonológica. A seguir, analisamos a relação de cada um destes aspectos com a aprendizagem da matemática. 
Resgate Lexical. O componente de velocidade de nomeação relaciona-se com a hipótese de Geary (1993) de que déficits na representação e recordação de informações semânticas da memória de longo prazo estariam relacionados à comorbidade entre a discalculia e a dislexia. O resgate lexical influencia a fluência de leitura e de cálculos, uma vez que essas habilidades exigem o resgate apropriado de sons ou números e é uma medida da qualidade das representações fonológicas de longo prazo. Além disso, o resgate eficiente de códigos fonológicos permite que as crianças desloquem recursos atencionais para procedimentos de cálculos (Bull \& Johnston, 1997; Geary, 1993).

Em um estudo com crianças com dificuldades específicas de linguagem (Specific Learning Impairment - SLI; Koponen, Mononen, Räsänen, \& Ahonen, 2006), a velocidade de resgate lexical foi a única variável linguística que explicou a diferença entre o grupo SLI e controles em tarefas numéricas que tinham algum componente verbal, como a habilidade de resgatar a resposta de fatos aritméticos.

Em relação à especificidade dos déficits em tarefas de nomeação seriada rápida (RAN), Willburger, Fussenegger, Moll, Wood e Landerl (2008) encontraram um padrão inespecífico em crianças disléxicas que foram mais lentas em todas as condições de nomeação (dígitos, letras e quantidades). Já as crianças com discalculia apresentaram um déficit específico de domínio: foram mais lentas apenas na nomeação de quantidades. Esse achado sugere que os discalcúlicos não apresentam dificuldades proeminentes na transcodificação arábico-verbal, já que obtiveram desempenho dentro da média na nomeação de dígitos. O grupo duplo-déficit, por sua vez, apresentou um padrão de déficits aditivos.

Em contrapartida, Pauly et al. (2011) encontraram déficits específicos de domínio em tarefas de RAN: crianças em risco de desenvolver problemas de leitura apresentaram déficits na nomeação de letras e objetos; crianças em risco de desenvolver problemas de matemáticas apresentaram déficits na nomeação de pontos e dígitos, e o grupo com risco para ambos os do- mínios apresentou um padrão aditivo de déficits. Os próprios autores sugerem que a divergência em relação aos achados anteriores pode estar relacionada com o critério de seleção de grupos: as crianças com risco de desenvolver problemas de leitura foram selecionadas a partir do desempenho em tarefas de processamento fonológico, o que pode ter contribuído para que grupos com outras dificuldades de linguagem não tenham sido incluídos na amostra.

Memória de Trabalho e Memória de Curto-Prazo Fonológica. De acordo com o modelo inicial de memória de trabalho proposto por Baddeley e Hitch (1974), o executivo central seria responsável pela alocação de recursos atencionais e manipulação de informação; e existiriam dois buffers, de capacidade limitada, que armazenam formas distintas de conteúdos. O buffer viso-espacial mantém informações de caráter viso-espacial e se relaciona com representações posteriores de semântica visual da memória de longo prazo. A alça fonológica (aqui também descrita como 'memória fonológica') é o aspecto do sistema memória de trabalho responsável pela codificação e armazenamento de informações verbais ou sonoras.

Ao realizar operações aritméticas, a criança deve manter na memória, por exemplo, "um mais um é igual a..." enquanto realiza o problema. A eficiência da memória de trabalho fonológica também pode influenciar o resgate de fatos aritméticos da memória de longo prazo.

De acordo com Rasmussen e Bisanz (2005) o buffer fonológico, medido pela tarefa de Dígitos do WISC (ordem direta) correlaciona-se com o desempenho de crianças escolares em todos os tipos de problemas aritméticos (verbais e não-verbais, com ou sem informações irrelevantes). As crianças pré-escolares, em contrapartida, apresentam dificuldades na resolução de problemas aritméticos verbais e seu desempenho em problemas não-verbais correlaciona-se com a memória de trabalho viso-espacial, medida pela ordem direta do Cubos de Corsi. Uma crítica possível a esse artigo é que os autores utilizaram apenas a ordem direta do Cubos de Corsi e Dígitos como medidas de memória de trabalho, 
as quais não envolvem manipulação on-line de informação, e, consequentemente, poderiam ser melhor consideradas medidas de memória de curto prazo. Neste estudo, a ordem inversa da tarefa Dígitos foi utilizada como uma medida fonológica do executivo central e uma tarefa de Counting Span como medida visual do executivo central e o mesmo padrão foi encontrado: o executivo central fonológico se correlacionaria com o desempenho das crianças mais velhas e o visual, com o de crianças pré-escolares.

McKenzie, Bull e Gray (2003) corroboram a sequência desenvolvimental proposta por Rasmussen e Bisanz (2005). Os autores utilizaram testes de interferência fonológica e viso-espacial durante a realização de tarefas aritméticas e concluíram que crianças mais novas (entre $6 \mathrm{e}$ 7 anos) dependem menos de representações fonológicas para a realização de cálculos comparadas com crianças mais velhas (8 e 9 anos), as quais utilizariam estratégias fonológicas e viso-espaciais.

Meyer, Salimpoo, Wu, Geary e Menon (2010), investigaram os aspectos específicos da memória de trabalho que influenciam no processamento numérico básico, resolução de cálculos e problemas matemáticos em crianças de $2^{\mathrm{a}} \mathrm{e}$ $3^{\text {a }}$ séries. Segundo os autores, os componentes da memória de trabalho que influenciam no desempenho da matemática variam ao longo do desenvolvimento: o executivo central e o buffer fonológico seriam mais importantes no aprendizado inicial, para o aprendizado da mudança de códigos verbais em formato numérico e, em estágios posteriores, a memória de trabalho viso-espacial seria o subcomponente fundamental. Uma possível fonte de divergência em relação ao modelo de Rasmussen e Bisanz (2005) pode se dever ao fato de que as crianças neste estudo eram mais velhas e, além disso, o subteste de problemas matemáticos utilizados por Meyer et al. (2010) continha itens de identificação de formas geométricas e interpretação de gráficos, o que pode estar relacionado com a influência do processamento viso-espacial em crianças mais velhas. Segundo os autores, esses achados sugerem uma mudança da circuitaria pré-frontal para circuitos mediados pelo córtex parietal, ao longo do aprendizado da matemática.

Consciência Fonológica. A habilidade de percepção e manipulação dos sons da língua prediz o desempenho da leitura e estudos recentes sugerem a importância desse componente do aprendizado da matemática. A consciência fonológica pode ser investigada através de tarefas que exigem a distinção dos sons que formam as palavras; como detecção de rimas e junção de sons isolados para criar palavras (Lewkowicz, 1980). De acordo com Castles e Coltheart (2004), a medida de consciência fonológica que apresenta maior correlação com o aprendizado da leitura e escrita é a consciência fonêmica, a qual envolve análise, manipulação e síntese de fonemas isolados e sua relação com a palavra como um todo.

Leather e Henry (1994) encontraram que $31 \%$ da variância do desempenho aritmético em crianças de 7 anos é explicada pela consciência fonêmica (escore compósito das seguintes tarefas: supressão de fonemas e seqüenciamento de fonemas para formar uma palavra). De acordo com os autores, a natureza da associação entre consciência fonológica e matemática está relacionada ao fato que manipulações fonológicas exigem processos aritméticos (por exemplo, tarefas de supressão de fonemas exigem, literalmente, a subtração de um som) e envolvem memória de trabalho. Um problema metodológico deste artigo é o fato que o desempenho da matemática foi medido apenas através do subteste de Aritmética do WISC-R, o qual faz parte da escala de QI verbal e depende, marcadamente, do processamento para a manutenção e resolução do problema e articulação da reposta.

Em um estudo com crianças típicas de $4^{\mathrm{a}} \mathrm{e}$ $5^{\mathrm{a}}$ séries, De Smedt et al. (2010) encontraram que uma medida de consciência fonêmica (tarefa de supressão de fonemas) estava relacionada especificamente com operações aritmética envolvendo a manipulação de pequenas magnitudes, que possuíam resultados menores que 25. Além disso, a consciência fonêmica estava associada com esses cálculos (adição, multiplicação e subtração) mesmo após controlar ha- 
bilidade de leitura e memória de curto-prazo fonológica.

Hecht, Torgesen, Wagner, e Rashotte (2001) apontam que a consciência fonológica prediz o desempenho da matemática em crianças de idade escolar. Um dos objetivos principais deste estudo foi avaliar a relação específica dos diversos componentes do processamento fonológico com o desenvolvimento da aprendizagem da matemática. No intervalo entre $3^{\mathrm{a}}$ e $4^{\mathrm{a}}$ série e entre $4^{\mathrm{a}}$ e $5^{\mathrm{a}}$ série, a consciência fonológica foi a única medida do processamento fonológico que explicava parte da variância no desempenho em um teste normatizado de aritmética $(6 \%$ da variância e $2 \%$ da variância, respectivamente), mesmo com o controle da inteligência e conhecimento prévio de matemática. A memória fonológica e velocidade de processamento seriam influentes apenas em crianças mais novas, entre a $2^{\mathrm{a}}$ e $3^{\mathrm{a}}$ série. Segundo os autores, os mesmos recursos da memória de trabalho que são utilizados na resolução de problemas matemáticos também são recrutados em tarefas de consciência fonológica.

Simmons, Singleton e Horne (2008) demonstraram que a relação entre a consciência fonológica e o aprendizado da matemática foi independente de medidas de vocabulário e raciocínio não-verbal. Neste estudo longitudinal, os autores demonstraram que a consciência fonológica (medida através de uma tarefa de detecção de rimas) e a memória viso-espacial (medida pela tarefa "Rabbits", versão computadorizada e lúdica da ordem direta do Cubos de Corsi) são capazes de predizer o desempenho na aritmética em crianças de 6 anos e apenas a consciência fonológica pode prever, isoladamente, o desempenho na leitura. A medida de desempenho artimético utlizada neste estudo é de validade questionável, uma vez que é constituída por 34 problemas oralmente formulados, acompanhados de figuras (ex: Cada cavalo precisa de sapatos novos. Quantos sapatos o fazendeiro precisa comprar ao todo?). Este tipo de tarefa exige manipulação verbal e viso-espacial de informação e não avalia outros aspectos importantes da aritmética, como a automatização de fatos, representação de magnitudes e valor posicional.
É interessante notar que, mesmo em crianças mais novas, como no estudo de Simmons et al. (2008), o processamento fonológico influencia a aprendizagem da matemática e da leitura, mesmo com o controle da inteligência e vocabulário.

Os estudos descritos acima utilizam medidas de aspectos diferentes da consciência fonológica (detecção de rimas, supressão de fonemas), e do processamento numérico (testes padronizados, tarefas experimentais de cálculos) o que dificulta a generalização dos achados.

\section{O Papel do Processamento Fonológico no Desenvolvimento da Aprendizagem Matemática}

O modelo desenvolvimental de Rasmussen e Bisanz (2005) sugere que o processamento viso-espacial é essencial para a aprendizagem da matemática para crianças em idade pré-escolar, as quais representam quantidades através de 'tokens'. O processamento fonológico, especificamente a memória fonológica, é mais influente nos anos seguintes, à medida que começam a existir representações verbais de quantidade durante o processo de escolarização formal; e o processamento viso-espacial perderia sua influência, por volta dos 6 anos de idade. Os autores avaliaram 34 pré-escolares e 29 crianças de primeira série que resolveram problemas aritméticos verbais (lidos pelo examinador) e não-verbais (com a manipulação de fichas). As crianças pré-escolares tiveram desempenho superior nos problemas não-verbais e não houve diferença entre os dois tipos de problema nas crianças de $1^{\text {a }}$ série. Em crianças pré-escolares, o escore na tarefa de Cubos de Corsi foi o melhor preditor do desempenho nos problemas não-verbais e a tarefa de Counting Span - medida do executivo central - nos problemas verbais. Já em crianças de $1^{a}$ série, o desempenho na tarefa de Digit Span (ordem direta e inversa) foi o melhor preditor no desempenho dos problemas verbais e nenhuma das medidas de memória de trabalho explicou porcentagem significativa da variância nos problemas não-verbais. Os autores justificam este achado devido ao fato que $24 \%$ das crianças de $1^{a}$ série desenvolveram estratégias externalizantes exclusivamente pra resolução de proble- 
mas não-verbais como, por exemplo, representar magnitudes nos dedos, o que diminuiria a carga da memória de trabalho.

Por outro lado, o modelo proposto por Krajewski e Schneider (2009) tem como um de seus pressupostos a hipótese de palavras numéricas isoladas (isolated number words hypothesis), segundo a qual a consciência fonológica não influenciaria tarefas que exigem a manipulação de quantidades, mas apenas tarefas em que as palavras que representam números são isoladas da representação de quantidade. O processamento fonológico seria importante, especificamente, no aprendizado das palavras referentes aos números, e seu seqüenciamento. Dessa forma, sua influência antecederia a ligação da palavra numérica com a quantidade que ela representa, processo que, por sua vez, dependeria do aprendizado do princípio de cardinalidade e memória viso-espacial. Os autores acompanharam 91 crianças da pré-escola até a terceira série. Na fase pré-escolar, as crianças realizaram testes relacionados ao sequenciamento de palavras numéricas, transcodificação arábico-verbal, comparação de magnitudes não-simbólicas, correspondência palavra numérica-quantidade e comparação de magnitudes simbólicas (apresentadas oralmente); além de medidas de consciência fonológica (síntese de fonemas e detecção de rimas), memória de trabalho fonológica e viso-espacial (dígitos do WISC e Cubos de Corsi) e inteligência não-verbal. $\mathrm{Na}$ terceira série, elas foram avaliadas através de um teste padronizado de matemática e de escrita. Os padrões de correlação entre as medidas do processamento numérico e consciência fonológica foram similares entre a pré-escola e a terceira série $(r=0,47, r=0,48$, respectivamente). No modelo de equação estrutural, os autores encontraram que a consciência fonológica influenciava a aquisição de habilidades matemáticas mais básicas, como o nome das palavras numéricas ("um, dois, três"), enquanto a memória de trabalho viso-espacial está relacionada com a ligação posterior das palavras com as quantidades.

A ordenação temporal da participação do processamento fonológico e viso-espacial é, portanto, oposta ao sugerido pelo modelo de
Rasmussen e Bisanz (2005), o qual investigou especificamente crianças pré-escolares e de primeira série, enquanto Krajewski e Schneider (2009) fizeram um estudo longitudinal dos 5 aos 8 anos. Além disso, Rasmussen e Bisanz (2005) investigaram especificamente a memória fonológica e problemas aritméticos; enquanto Krajewski e Schneider também avaliaram a consciência fonológica e aspectos mais básicos da matemática; como contagem e comparação de magnitudes.

O modelo de Krajewski e Schneider (2009) confirma apenas parcialmente a hipótese de Simmons e Singleton (2008) em relação ao impacto das representações fonológicas em aspectos verbais da aritmética (weak phonological representation hypothesis) já que, neste modelo, déficits na representação fonológica causariam impacto negativo especificamente na aquisição de códigos numéricos verbais. Os déficits fonológicos não influenciaram habilidades numéricas mais sofisticadas ou posteriores, (apenas indiretamente, devido à automação da sequência numérica) mesmo quando há mediação verbal, como no caso de procedimentos de resolução de cálculos por estratégias verbais.

\section{Correlatos Anátomo-Funcionais}

Estudos de neuroimagem funcional sugerem que a porção horizontal do sulco intraparietal é ativada durante a execução de tarefas não-verbais de matemática, que envolvem a representação semântica de quantidades; e este seria o principal circuito ativado em tarefas de aritmética (Wilson \& Dehaene, 2007). No caso de crianças com discalculia, os resultados ainda são inconclusivos: Kaufmann et al. (2009) sugerem que haveria um aumento da ativação do sulco intraparietal, enquanto Kucian et al. (2011) mostram que crianças discalcúlicas têm diminuição da atividade nessa região. Kaufmann, Wood, Rubinsten e Henik (2011) realizaram uma meta-análise com 5 artigos publicados sobre ressonância magnética funcional em crianças com discalculia do desenvolvimento e/ou dificuldade de aprendizagem da matemática, em tarefas de processamento numérico simbólico e não-simbólico. Os autores utilizaram os mapas $A L E$, que 
permitem calcular a significância estatística de áreas consistentemente ativadas nos estudos. Os controles produziram maior ativação no pré-cuneo esquerdo e no lobo parietal inferior direito. Já as crianças com dificuldade de aprendizagem da matemática apresentaram maior ativação em áreas fronto-parietais bilaterais. Uma conclusão importante deste estudo é que as crianças com discalculia produzem uma maior ativação da porção anterior do sulco intraparietal em relação aos controles. Os autores sugerem que esta ativação anterior pode estar relacionada à estratégias compensatórias utilizadas pelas crianças com dificuldade de aprendizagem da matemática, como a contagem dos dedos.

Em relação à dislexia, foram encontradas diferenças na ativação de regiões occipitais e temporais no hemisfério esquerdo (Shaywitz \& Shaywitz, 2005) entre o grupo clínico e o controle durante a realização de tarefas com componente fonológico. Áreas ventrais e dorsais são hipo-ativadas em crianças pré-escolares que ainda não aprenderam a ler, como áreas temporo-parietais, occipto-temporais e o giro angular (Simos et al., 2002). Crianças com habilidades de leitura dentro da média apresentam maior ativação em áreas do hemisfério esquerdo, incluindo os giros: frontal inferior, temporal superior, parieto-temporal e em porções anteriores e posteriores do hemisfério direito.

$\mathrm{O}$ giro angular esquerdo pode ser considerado o lócus comum entre a matemática e a leitura. Programas de reabilitação da leitura baseados em estratégias fonológicas aumentam a ativação do giro angular esquerdo (Shaywitz et al., 2004). Além disso, ele está associado com representações linguísticas de símbolos numéricos, conceitos e procedimentos de cálculos, e recordação de fatos aritméticos (Dehaene et al., 2003; Rubinsten \& Henik, 2009).

Em um estudo de neuroimagem com adultos, Grabner et al. (2009) utilizaram uma medida de auto-relato para avaliar estratégias utilizadas na resolução de cálculos. Os autores encontraram maior ativação do giro angular esquerdo em itens de adição exata quando comparados com adição aproximada. Além disso, houve maior ativação em cálculos de multiplicação do que subtração. Os itens que foram solucionados por estratégias procedimentais tiveram maior ativação na circuitaria fronto-parietal, enquanto itens cujas respostas foram resgatadas da memória ativaram o giro angular esquerdo.

Price e Ansari (2011), também em estudo com adultos, encontraram ativação do giro angular esquerdo no processamento de algarismos arábicos, mas apenas durante apresentações longas $(500 \mathrm{~ms})$, que permitem algum tipo de percepção consciente. Houve maior ativação da porção dorsal do giro angular esquerdo, área relacionada com a identificação conceitual, o que indica que dígitos isolados contêm algum tipo de informação semântica. Não houve ativação desta área quando foram apresentados dígitos distorcidos ou letras.

\section{Processamento Fonológico e Aprendizagem da Aritmética em Crianças Com Transtornos do Desenvolvimento da Linguagem}

Sujeitos com dislexia apresentam déficits no processamento fonológico, portanto, a investigação de suas habilidades matemáticas permite a delimitação do papel destes prejuízos na matemática. Na última década, estudos com crianças e adultos que apresentam dificuldade específica da leitura e desempenho normal em testes padronizados de matemática demonstram que a investigação mais detalhada do processamento numérico de disléxicos, mesmo que com o senso numérico preservado, permite identificar prejuízos específicos da matemática.

Um aspecto da matemática que frequentemente tem sido associado ao processamento fonológico é o resgate de fatos aritméticos, principalmente os de adição e multiplicação, cuja resolução é mais dependente de estratégias verbais. Segundo Dehaene (1992), para resolver um problema matemático, a criança deve transformar os termos e o operador em um código verbal. A memorização da tabuada estaria relacionada à recordação do resultado na memória de longo prazo e, consequentemente, dependeria da qualidade das representações fonológicas de longo prazo. 
A maior parte dos estudos com disléxicos indica que a dificuldade na recordação de fatos aritméticos é o maior prejuízo na matemática deste grupo (Geary, Hamson, \& Hoard, 2000; Simmons \& Singleton, 2008).

Boets e De Smedt (2010) sugerem que, em crianças de 8 anos, essa dificuldade não é específica para o tipo de operação: o grupo com dislexia teve desempenho inferior aos controles em cálculos de multiplicação e subtração. A amostra de 13 disléxicos e 16 controles realizou uma tarefa experimental de cálculos, cujos itens de multiplicação poderiam ser solucionados por estratégias de recordação e os itens de subtração seriam procedimentais. É interessante notar que a falta de efeito de operação no grupo clínico sugere que os disléxicos não utilizaram estratégias eficientes de recordação dos fatos de multiplicação, já que é esperado que os problemas solucionados por estratégias de recordação sejam resolvidos de forma mais acurada e rápida comparados com os cálculos de subtração. Um problema metodológico neste artigo é que, apesar das crianças disléxicas não terem diagnóstico clínico de discalculia, elas apresentaram desempenho inferior no teste padronizado de matemática quando comparadas com os controles (teste exato de Fischer: $p<0,05$ ), o que dificulta excluir possíveis déficits no processamento numérico que influenciariam nos cálculos.

De Smedt et al. (2010) também não encontraram este efeito de especificidade do tipo de operação em crianças normais de quarta e quinta séries e sugerem que, é ao longo do desenvolvimento e principalmente na fase adulta, que os indivíduos passam a utilizar códigos e estratégias diferentes para resolver problemas de subtração, por exemplo.

Simmons e Singleton (2009) investigaram as potencialidades e dificuldades de crianças disléxicas, de 10 e 11 anos, no processamento numérico. Segundo os autores, disléxicos não apresentam dificuldades de contagem, apesar de essa habilidade ter, marcadamente, um componente verbal. $\mathrm{O}$ grupo clínico também apresentou compreensão normal do princípio do valor posicional. Uma crítica que pode ser realizada ao artigo é que a tarefa de valor posicional envolvia um componente de comparação de magnitude, não sendo, portanto, uma medida pura de transcodificação. Os disléxicos tiveram dificuldades na recordação de fatos aritméticos, o que sugere que os códigos fonológicos da memória de longo prazo estão prejudicados.

Göbel e Snowling (2010), a partir de estudo das habilidades aritméticas em adultos disléxicos, sugerem que as dificuldades verbais da aritmética podem ser consideradas outro endofenótipo da dislexia. Os participantes eram universitários e com inteligência normal (QI > 90) e apresentavam dificuldades restritas aos cálculos exatos. Não houve diferenças significativas no desempenho em tarefas de comparação de algarismos de dois dígitos, cálculos aproximados e julgamento de paridade entre disléxico e controles. As autoras sugerem uma dissociação no desempenho de cálculos aproximados e exatos em adultos disléxicos, o que indica que o prejuízo na matemática neste grupo clínico não sofre influência do processamento analógico de magnitudes, e se limita aos aspectos verbais da aritmética.

Aproximadamente $7 \%$ de crianças em idade escolar apresentam dificuldades específicas da linguagem (specific language impairments - SLI; Bishop, 1997). Estas crianças apresentam prejuízo na linguagem oral e inteligência não-verbal dentro ou superior à média (Cowan, Donlan, Newton, \& Lloyd, 2005). Apesar das dificuldades apresentadas por essas crianças serem, por definição, específicas na linguagem, o desempenho na matemática também é prejudicado (Donlan, 2007).

No que diz respeito aos déficits no processamento numérico, é possível hipotetizar que o desempenho destas crianças seja marcadamente inferior em aspectos verbais; como contagem, recordação de fatos aritméticos e transcodificação, e que as crianças não apresentem dificuldades em tarefas relacionadas à representação aproximada de magnitudes, como comparação e estimação numérica. Koponen et al. (2006) encontraram resultados divergentes: o grupo SLI, de crianças entre 9 e 11 anos, teve desempenho abaixo da média em tarefas matemáticas que não exigiam manipulação de informações ver- 
bais, como comparação de multidígitos e linha numérica e em tarefas que envolviam aspectos verbais, como adição. Segundo os autores, tal resultado pode ter ocorrido devido a diferenças entre o grupo SLI e os controles em relação ao raciocínio não verbal e a heterogeneidade do grupo clínico. É importante notar que as tarefas consideradas pelos autores como 'não-verbais' envolviam a manipulação do código simbólico, o qual pode estar relacionado com a linguagem à medida que envolve a ligação de um código com sua representação semântica e fonológica.

Donlan e Gourlay (1999) investigaram a noção de valor posicional e comparação de magnitudes em crianças SLI. O grupo clínico de 13 crianças de 8 anos foi comparado com dois grupos controles: um foi pareado por idade e outro por habilidades linguísticas. As crianças com SLI acertaram $100 \%$ dos itens de comparação de magnitude quando os estímulos eram números de um dígito, sendo este desempenho superior ao do grupo controle pareado por nível de linguagem. $\mathrm{Na}$ tarefa de comparação de algarismos multidígitos, o grupo SLI também teve desempenho de acordo com o esperado para sua faixa etária.

Cowan et al. (2005) investigaram o processamento numérico de crianças SLI entre 7 e 9 anos de idade com o objetivo de investigar as especificidades das dificuldades da matemática dessas crianças. As correlações entre os testes de matemática, como contagem e transcodificação, com as tarefas de compreensão oral de linguagem foram maiores que as correlações das tarefas aritméticas com a memória de trabalho (medida por uma tarefa de repetição de pseudopalavras). O grupo SLI que estudava em escola regular teve desempenho abaixo dos controles da mesma faixa etária nas tarefas de contagem, cálculos, problemas matemáticos e comparação de magnitudes simbólicas. Entretanto, este estudo apresenta alguns problemas metodológicos: a tarefa de transcodificação continha apenas 8 itens; cálculos e problemas foram apresentados oralmente, o que pode ter causado uma sobrecarga da memória de trabalho fonológica das crianças. Além disso, os autores não controlaram a habilidade de leitura dos controles. Apesar de habilidades de linguagem oral e escrita serem correlacionadas, é importante investigar a influência específica de cada uma no desempenho da matemática de crianças SLI.

É necessário que esse grupo clínico seja melhor investigado em pesquisas relacionadas às habilidades matemáticas com tarefas não-simbólicas para que o papel da linguagem no processamento numérico seja melhor delimitado.

\section{Conclusão}

Os estudos revisados demonstram que déficits no processamento fonológico são fator de risco para dificuldades de leitura e matemática. Sua importância já é bem demarcada na pesquisa de dislexia, mas o interesse do papel deste mecanismo cognitivo na aprendizagem da aritmética é relativamente recente.

Alguns achados ainda são inconclusivos, devido às diferenças metodológicas entre os estudos, que dificultam a comparação dos dados. Existem poucas medidas de processamento fonológico que são padronizadas. Os pesquisadores então, se veem obrigados a desenvolver tarefas experimentais que não podem ser comparadas entre si e, frequentemente, medem aspectos diferentes do processamento fonológico (consciência fonêmica, percepção de rimas).

Além disso, nenhum estudo controlou, simultaneamente, os mecanismos cognitivos candidatos a influenciar o processamento numérico como a memória de trabalho, senso numérico, processamento viso-espacial e consciência fonológica. Deste modo, é difícil controlar a influência específica de cada um deles.

Apesar destas lacunas, o presente artigo demonstrou que diversos estudos apoiam a hipótese de mecanismos compartilhados na aprendizagem da leitura e da matemática. De acordo com o modelo de déficits múltiplos proposto por Pennington (2006), a comorbidade entre dois transtornos está relacionada ao compartilhamento de fatores etiológicos e mecanismos cognitivos. No caso da discalculia e dislexia, a partir dos estudos revisados, podemos sugerir que o giro angular esquerdo é o lócus comum à aprendizagem habilidades de leitura e escrita e que o processamento fonológico é o principal mecanismo 
cognitivo cujo déficit pode causar impacto em ambos os transtornos. Déficits no processamento fonológico são, então, condição suficiente, mas não necessária, para dificuldades de aprendizagem da matemática.

É importante que crianças com dislexia ou dificuldades específicas de linguagem também recebam intervenções voltadas ao processamento numérico, especialmente no que diz respeito à memorização da tabuada e interpretação dos problemas matemáticos.

\section{Referências}

American Psychiatric Association. (2000). Diagnostic and statistical manual of mental disorders: $D S M I V$ ( $4^{\text {th }}$ ed.). Washington, DC: Author.

Baddeley, A. D., \& Hitch, G. (1974). Working memory. In G. A. Bower (Ed.), The psychology of learning and motivation (pp. 47-89). New York: Academic Press.

Bishop, D. V. M. (1997). Uncommon understanding: Development and disorders of language comprehension in children. Hove, UK: Psychology Press.

Boets, B., \& De Smedt, B. (2010). Single-digit arithmetic in children with dyslexia. Dyslexia, 16(2), 183-191. doi:10.1002/dys.403

Bowers, P. G., \& Wolf, M. (1993). Theoretical links among naming speed, precise timing mechanisms and orthographic skill in dyslexia. Reading and Writing, 5(1), 69-85. doi:10.1007/ BF01026919

Bull, R., \& Johnston, R. S. (1997). Children's arithmetical difficulties: Contributions from processing speed, item identification, and short-term memory. Journal of Experimental Child Psychology, 65(1), 1-24. doi:10.1006/jecp.1996.2358

Butterworth, B. (2005). The development of arithmetical abilities. Journal of Child Psychology and Psychiatry and Allied Disciplines, 46(1), 3-18. doi:10.1111/j.1469-7610.2004.00374.x

Castles, A., \& Coltheart, M. (2004). Is there a causal link from phonological awareness to success in learning to read? Cognition, 91(1), 77-111. doi:10.1016/S0010-0277(03)00164-1

Costa, A. J., Silva, J. B. L., Pinheiro-Chagas, P., Krinzinger, H., Lonnemann, J., Willmes, K., ...Haase, V. G. (2011). A hand full of numbers:
A role for offloading in arithmetics learning? Frontiers in Cognition, 2, 1-12. doi:10.3389/fpsyg.2011.00368

Cowan, R., Donlan, C., Newton, E., \& Lloyd, D. (2005). Number skills and knowledge in children with specific language impairment. Journal of Educational Psychology, 97(4), 732-744. doi:10.1037/0022-0663.97.4.732

De Smedt, B., Taylor, J., Archibald, L., \& Ansari, D. (2010). How is phonological processing related to individual differences in children's arithmetic skills? Developmental Science, 13, 508-520. doi:10.1111/j.1467-7687.2009.00897.x

Dehaene, S. (1992). Varieties of numerical abilities. Cognition, 44, 1-42. doi:10.1016/0010$-0277(92) 90049-\mathrm{N}$

Dehaene, S., \& Cohen, L. (1995). Towards an anatomical and functional model of number processing. Mathematical Cognition, 1, 83-120.

Dehaene, S., Piazza, M., Pinel, P., \& Cohen, L. (2003). Three parietal circuits for number processing. Cognitive Neuropsychology, 20, 487506. doi:10.1080/02643290244000239

Donlan, C. (2007). Mathematical development in children with specific language impairments. In D. B. Berch \& M. M. M. Mazzocco (Eds.), Why is math so hard for some children? (pp. 151172). Baltimore, MD: Brooks.

Donlan, C., \& Gourlay, S. (1999). The importance of non-verbal skills in the acquisition of place-value knowledge: Evidence from normally-developing and language-impaired children. British Journal of Developmental Psychology, 17(1), 1-19. doi:10.1348/026151099165113

Fawcett, A. J., \& Nicolson, R. I. (2001). Dyslexia: The role of the cerebellum. In A. J. Fawcett (Ed.), Dyslexia theory and good practice (pp. 192-214). London: Whurr.

Geary, D. C. (1993). Mathematical disabilities: Cognitive, neuropsychological, and genetic components. Psychological Bulletin, 114(2), 345-362. doi:10.1016/j.lindif.2009.10.008

Geary, D. C. (2000). From infancy to adulthood: The development of numerical abilities. European Child Adolescent Psychiatry, 2(22), II11-I16. doi:10.1007/s007870070004

Geary, D. C. (2010). Mathematical disabilities: Reflections on cognitive, neuropsychological, and genetic components. Learning and Individu- 
al Differences, 20(2), 130. doi:10.1016/j.lindif.2009.10.008

Geary, D. C., Hamson, C. O., \& Hoard, M. K. (2000). Numerical and arithmetical cognition: A longitudinal study of process and concept deficits in children with learning disability. Journal of Experimental Child Psychology, 77(3), 236-263. doi:10.1006/jecp.2000.2561

Geary, D. C., Hoard, M. K., Byrd-Craven, J., \& DeSoto, M. C. (2004). Strategy choices in simple and complex addition: Contributions of working memory and counting knowledge for children with mathematical disability. Journal of Experimental Child Psychology, 88(2), 121-151. doi:10.1016/j.jecp.2004.03.002

Girelli, L., \& Seron, X. (2001). Rehabilitation of number processing and calculation skills. Aphasiology, 15(7), 695-712. doi:10.1080/02687040143000131

Göbel, S. M., \& Snowling, M. J. (2010). Number-processing skills in adults with dyslexia. The Quarterly Journal of Experimental Psychology, 63(7), 1361-1373. doi:10.1080/17470210903359206

Grabner, R. H., Ansari, D., Koschutnig, K., Reishofer, G., Ebner, F., \& Neuper, C. (2009). To retrieve or to calculate? Left angular gyrus mediates the retrieval of arithmetic facts during problem solving. Neuropsychologia, 47(2), 604-608.

Halberda, J., Mazzocco, M. M., \& Feigenson, L. (2008). Individual differences in non-verbal number acuity correlate with maths achievement. Nature, 455, 665-668. doi:10.1038/nature07246

Hecht, S. A., Torgesen, J. K., Wagner, R. K., \& Rashotte, C. A. (2001). The relations between phonological processing abilities and emerging individual differences in mathematical computation skills: A longitudinal study from second to fifth grades. Journal of Experimental Child Psychology, 79(2), 192-227. doi:10.1006/ jecp.2000.2586

Huttenlocher, J., Jordan, N. C., \& Levine, S. C. (1994). A mental model for early arithmetic. Journal of Experimental Psychology: General, 123(3), 284-296. doi:10.1037/0012-1649.28.4.644

Jordan, N. C. (2007). Do words count? Connections between reading and mathematics difficulties. In D. B. Berch \& M. M. M. Mazzocco (Eds.), Why is math so hard for some children (pp. 107-120). Baltimore, MD: Brooks.
Kaufmann, L., Vogel, S. E., Starke, M., Kremser, C., Schocke, M., \& Wood, G. (2009). Developmental dyscalculia: Compensatory mechanisms in left intraparietal regions in response to nonsymbolic magnitudes. Behavioral and Brain Functions, 5(1), 35. doi:10.1186/1744-9081-5-35

Kaufmann, L., Wood, G., Rubinsten, O., \& Henik, A. (2011). Meta-analyses of developmental fMRI studies investigating typical and atypical trajectories of number processing and calculation. $D e-$ velopmental Neuropsychology, 36(6), 763-787. doi:10.1080/87565641.2010.549884

Koponen, T., Mononen, R., Räsänen, P., \& Ahonen, T. (2006). Basic numeracy in children with specific language impairment: Heterogeneity and connections to language. Journal of Speech, Language and Hearing Research, 49(1), 58-73. doi:10.1044/1092-4388(2006/005

Krajewski, K., \& Schneider, W. (2009). Exploring the impact of phonological awareness, visual-spatial working memory, and preschool quantity-number competencies on mathematics achievement in elementary school: Findings from a 3-year longitudinal study. Journal of Experimental Child Psychology, 103(4), 516-531. doi:10.1016/j.jecp.2009.03.009

Kucian, K., Grond, U., Rotzer, S., Henzi, B., Schönmann, C., Plangger, F., ...von Aster, M. (2011). Mental number line training in children with developmental dyscalculia. Neuroimage, 57(3), 782-795. doi:10.1016/j.neuroimage.2011.01.070

Landerl, K., Bevan, A., \& Butterworth, B. (2004). Developmental dyscalculia and basic numerical capacities: A study of 8-9-year-old students. Cognition, 93(2), 99-125. doi:10.1016/j.cognition.2003.11.004

Landerl, K., Fussenegger, B., Moll, K., \& Willburger, E. (2009). Dyslexia and dyscalculia: Two learning disorders with different cognitive profiles. Journal of Experimental Child Psychology, 103, 309-324. doi:10.1016/j.jecp.2009.03.006

Landerl, K., \& Moll, K. (2010). Comorbidity of learning disorders: Prevalence and familial transmission. Journal of Child Psychology and Psychiatry and Allied Disciplines, 51(3), 287294. doi:10.1111/j.1469-7610.2009.02164.x

Leather, C. V., \& Henry, L. A. (1994). Working memory span and phonological awareness tasks as predictors of early reading ability. Journal of 
Experimental Child Psychology, 58(1), 88-111. doi:10.1348/026151005X26804

Lee, J. (2009). Universals and specifics of math self-concept, math self-efficacy, and math anxiety across 41 PISA 2003 participating countries. Learning and Individual Differences, 19(3), 355365. doi:10.1016/j.lindif.2008.10.009

LeFevre, J. A., Fast, L., Skwarchuk, S. L., Smith-Chant, B. L., Bisanz, J., Kamawar, D., \& Penner-Wilger, M. (2010). Pathways to mathematics: Longitudinal predictors of performance. Child Development, 8, 1753-1767. doi:10.1111/ j.1467-8624.2010.01508.x

Lewis, C., Hitch, G. J., \& Walker, P. (1994). The prevalence of specific arithmetic difficulties and specific reading difficulties in 9- to 10-year-old boys and girls. Journal of Child Psychology and Psychiatry, 35, 283-292. doi:10.1111/j.1469-7610.1994.tb01162.x

Lewkowicz, N. K. (1980). Phonemic awareness training: What to teach and how to teach it. Journal of Educational Psychology, 72(5), 686-700.

Mckenzie, B., Bull, R., \& Gray, C. (2003). The effects of phonological and visual-spatial interference on children's arithmetical performance. Educational and Child Psychology, 20(3), 93-108.

Meyer, M. L., Salimpoor, V. N., Wu, S. S., Geary, D. C., \& Menon, V. (2010). Differential contribution of specific working memory components to mathematics achievement in $2^{\text {nd }}$ and $3^{\text {rd }}$ graders. Learning and Individual Differences, 20(2), 101-109. doi:10.1016/j.lindif.2009.08.004

Mussolin, C., Mejias, S., \& Noël, M.-P. (2010). Symbolic and nonsymbolic number comparison in children with and without dyscalculia. Cognition, 115(1), 10-25. doi:10.1016/j.cognition.2009.10.006

Nicolson, R. I., \& Fawcett, A. J. (2001). Dyslexia as a learning disability. In A. J. Fawcett (Ed.), Dyslexia: Theory and good practice (pp. 141-160). London: Whurr.

Noël, M.-P. (2009). Counting on working memory when learning to count and to add: A preschool study. Developmental Psychology, 45(6), 16301643. doi:10.1037/a0016224

Pauly, H., Linkersdörfer, J., Lindberg, S., Woerner, W., Hasselhorn, M., \& Lonnemann, J. (2011). Domain-specific Rapid Automatized Naming deficits in children at risk for learning disabi- lities. Journal of Neurolinguistics, 24(5), 602610. doi:10.1016/j.jneuroling.2011.02.002

Pennington, B. F. (2006). From single to multiple deficit models of developmental disorders. Cognition, 101(2), 385-413. doi:10.1016/j.cognition.2006.04.008

Piazza, M., Facoetti, A., Trussardi, A. N., Berteletti, I., Conte, S., Lucangeli, D., ...Zorzi, M. (2010). Developmental trajectory of number acuity reveals a severe impairment in developmental dyscalculia. Cognition, 116, 33-41. doi:10.1016/j. cognition.2010.03.012

Price, G. R., \& Ansari, D. (2011). Symbol processing in the left angular gyrus: Evidence from passive perception of digits. NeuroImage, 57(3), 12051211. doi:10.1016/j.neuroimage.2011.05.035

Rasmussen, C., \& Bisanz, J. (2005). Representation and working memory in early arithmetic. Journal of Experimental Child Psychology, 91(2), 137-157. doi:10.3201/eid1105.040934

Raghubar, K. P., Barnes, M. A., \& Hecht, S. (2010). Working memory and mathematics: A review of developmental, individual difference, and cognitive approaches. Learning and Individual Differences, 20, 110-122. doi:10.1016/j.lindif.2009.10.005

Rourke, B. P., \& Conway, J. A. (1997). Disabilities of arithmetic and mathematical reasoning: Perspectives from neurology and neuropsychology. Journal of Learning Disabilities, 30(1), 34-46. doi:10.1177/002221949302600402

Rubinsten, O., \& Henik, A. (2006). Double dissociation of functions in developmental dyslexia and dyscalculia. Journal of Educational Psychology, 98(4), 854-867. doi:10.1037/00220663.98.4.854

Rubinsten, O., \& Henik, A. (2009). Developmental dyscalculia: Heterogeneity might not mean different mechanisms. Trends in Cognitive Sciences, 13, 92-99. doi:10.1016/j.tics.2008.11.002

Shalev, R. S., Auerbach, J., Manor, O., \& Gross-Tsur, V. (2000). Developmental dyscalculia: Prevalence and prognosis. European Child Adolescent Psychiatry, 9(Suppl. 2), S58-S64. doi:10.1007/ s007870070009

Shalev, R. S., Manor, O., \& Gross-Tsur, V. (1997). Neuropsychological aspects of developmental dyscalculia. Mathematica Cognition, 3, 105120. doi:10.1080/135467997387434 
Shaywitz, S. E., \& Shaywitz, B. A. (2005). Dyslexia (Specific Reading Disability). Biological Psychiatry, 57, 1301-1309. doi:10.1016/j.biopsych.2005.01.043

Shaywitz, B. A., Shaywitz, S. E., Blachman, B. A., Pugh, K. R., Fulbright, R. K., Skudlarski, P., ...Gore, J. C. (2004). Development of left occipitotemporal systems for skilled reading in children after a phonologically- Based intervention. Biological Psychiatry, 55(9), 926-933. doi:10.1016/j.biopsych.2003.12.019

Simmons, F. R., \& Singleton, C. (2008). Do weak phonological representations impact on arithmetic development? A review of research into arithmetic and dyslexia. Dyslexia, 14(2), 77-94. doi:10.1002/dys.341

Simmons, F. R., \& Singleton, C. (2009). The mathematical strengths and weaknesses of children with dyslexia. Journal of Research in Special Educational Needs, 9(3), 154-163. doi:10.1111/ j.1471-3802.2009.01128.x

Simmons, F., Singleton, C., \& Horne, J. (2008). Phonological awareness and visual-spatial sketchpad functioning predict early arithmetic attainment: Evidence from a longitudinal study. European Journal of Cognitive Psychology, 20, 711-722. doi:10.1080/09541440701614922

Simos, P. G., Fletcher, J. M., Bergman, E., Breier, J. I., Foorman, B. R., Castillo, E. M., ...Fitzgerald, M. (2002). Dyslexia-specific brain activation profile becomes normal following successful remedial training. Neurology, 58(8), 1203-1213. doi:10.1212/WNL.58.8.1203

Snowling, M. J., \& Hulme, C. (1994). The development of phonological skills. Philosophical Transactions of the Royal Society Series B, 346, 2128. doi:10.1098/rstb.1994.0124

Tallal, P., Miller, S. L., Jenkins, W. M., \& Merzenich, M. M. (1997). The role of temporal processing in developmental language-based learning disorders: Research and clinical implications. In B. Blachman (Ed.), Foundations of reading acquisition and dyslexia implications for early intervention (Vol. 3, pp. 49-66). Mahwah, NJ: Lawrence Erlbaum.

Tressoldi, P. E., Rosati, M., \& Lucangeli, D. (2007). Patterns of developmental dyscalculia with or without dyslexia. Neurocase, 13, 217-225.

Vellutino, F. R., Fletcher, J. M., Snowling, M. J., \& Scanlon, D. M. (2004). Specific reading disabi- lity (dyslexia): What have we learned in the past four decades? Journal of Child Psychology and Psychiatry and Allied Disciplines, 45(1), 2-40. doi:10.1046/j.0021-9630.2003.00305.x

Wagner, R. K., \& Torgesen, J. K. (1987). The nature of phonological processing and its causal role in the acquisition of reading skills. Psychological Bulletin, 101(2), 192-212. doi:10.1037//00332909.101.2.192

Willburger, E., Fussenegger, B., Moll, K., Wood, G., \& Landerl, K. (2008). Naming speed in dyslexia and dyscalculia. Learning and Individual Differences, 18(2), 224-236. doi:10.1016/j.lindif.2008.01.003

Wilson, A. J., \& Dehaene, S. (2007). Number sense and developmental dyscalculia. In D. Coch, G. Dawson, \& K. Fischer (Eds.), Human behavior, learning, and the developing brain: Atypical development (pp. 212-238). New York: Guilford.

Wimmer, H. (2006). Don't neglect reading fluency! Developmenta Science, 9(5), 447-448. doi:10.1111/j.1467-7687.2006.00527.x

Wimmer, H., \& Schurz, M. (2010). Dyslexia in regular orthographies: Manifestation and causation. Dyslexia, 16, 283-299. doi:10.1002/dys.411

Wolf, M., \& Bowers, P. G. (1999). The Double-Deficit Hypothesis for developmental dyslexias. Journal of Educational Psychology, 91, 1-24.

Wolf, M., Bowers, P. G., \& Biddle, K. (2000). Naming-speed processes, timing, and reading: A conceptual review. Journal of Learning Disabilities, 33(4), 387-407. doi:10.1177/002221940003300409

Zamarian, L., Ischebeck, A., \& Delazer, M. (2009). Neuroscience of learning arithmetic--Evidence from brain imaging studies. Neuroscience \& Biobehavioral Reviews, 33(6), 909-925. doi:10.1016/j.neubiorev.2009.03.005

Zuber, J., Pixner, S., Moeller, K., \& Nuerk, H. C. (2009). On the language specificity of basic number processing: Transcoding in a language with inversion and its relation to working memory capacity. Journal of Experimental Child Psychology, 102, 60-77. doi:10.1016/j. jecp.2008.04.003

Recebido: 04/10/2012

$1^{a}$ revisão: 04/05/2014

Aceite final: 20/07/2014 